\title{
EFFECTIVE $p$-ADIC BOUNDS FOR SOLUTIONS OF HOMOGENEOUS LINEAR DIFFERENTIAL EQUATIONS
}

BY

\author{
B. DWORK AND P. ROBBA
}

Dedicated to K. Iwasawa

\begin{abstract}
We consider a finite set of power series in one variable with coefficients in a field of characteristic zero having a chosen nonarchimedean valuation. We study the growth of these series near the boundary of their common "open" disk of convergence. Our results are definitive when the wronskian is bounded. The main application involves local solutions of ordinary linear differential equations with analytic coefficients. The effective determination of the common radius of convergence remains open (and is not treated here).
\end{abstract}

Let $K$ be an algebraically closed field of characteristic zero complete under a nonarchimedean valuation with residue class field of characteristic $p$. Let $D=$ $d / d x$

$$
L=D^{n}+C_{n-1} D^{n-1}+\cdots+C_{0}
$$

be a linear differential operator with coefficients meromorphic in some neighborhood of the origin. Let

$$
u=a_{0}+a_{1} x+\ldots
$$

be a power series solution of $L$ which converges in an open ( $p$-adic) disk of radius $r$. Our object is to describe the asymptotic behavior of $\left|a_{s}\right| r^{s}$ as $s \rightarrow \infty$. In a series of articles we have shown that subject to certain restrictions we may conclude that

$$
r^{s}\left|a_{s}\right|=O\left(s^{n-1}\right) \text {. }
$$

The object of the present article is to make this estimate effective. At the same time we greatly simplify, and generalize, our best previous results [12] for the noneffective form.

Our previous work was based on the notion of a generic disk together with a condition for reducibility of differential operators with unbounded solutions [4, Theorem 4]. Thus we transferred our given differential equation to a generic disk, reduced the problem there to the case of first order differential equations and then transferred the information back.

The present method eliminates the notion of generic disks and avoids the question of reducibility. We also avoid direct hypotheses on the coefficients of the differential equation. We impose conditions only on the solutions and on the wronskian. The main tools are the Frobenius formulation of the basis of the solution space (equation (3.7) below) and the boundary seminorm [10].

Received by the editors March 1, 1979 and, in revised form, April 27, 1979.

AMS (MOS) subject classifications (1970). Primary 12B40.

(c) 1980 American Mathematical Society $0002-9947 / 80 / 0000-0264 / \$ 05.75$ 
We show (Theorem 4.2 below) that (3) holds (with $r=1$ ) if $u$ lies in the $K$-span of $u_{1}, \ldots, u_{n}$ where each $u_{j}$ lies in $K[[x]]$, converges in $D\left(0,1^{-}\right)$, the open disk of radius unity and center at the origin provided the wronskian determinant of $u_{1}, \ldots, u_{n}$ is bounded on $D\left(0,1^{-}\right)$. The effective form is given by Theorem 4.3 and the case of apparent singularities is given in $\$ 5$. The method for extension to systems is given in $\$ 6$ which is simply an exposition of the known "equivalence" of linear systems with a single linear equation. In $\$ 7$ we indicate some pitfalls that must be avoided in giving a detailed discussion of systems.

We note that we have not completely responded to the original question, since the radii of convergence of the solutions of (1) at the origin need not all have the same radius of convergence. We have shown [6, (4.2.2), (4.2.6)] that in general such a situation causes no difficulty but the example of the third order differential operator

$$
\left(1 / x^{2}\right) D \circ(1-x) \circ D \circ x \circ D
$$

with solutions $1, \log x$ and $l_{2}(x)=\sum_{s=1}^{\infty} x^{s} / s^{2}$ shows that a finite dimensional function space with bounded wronskian may have a natural subspace which does not have a bounded wronskian. Furthermore the effective bounds (4.3.2) do not hold for the dilogarithm, $l_{2}(x)$ (but equation (3.6) with $n=3$ does hold for the third order linear differential equation satisfied by $\left.1, \log x, l_{2}(x)\right)$.

A further question (for details see $\S 8$ ) involves regular singularities. Here we suppose $F_{1}, \ldots, F_{n}$ are analytic on $D\left(0,1^{-}\right)$and that

$$
\left(u_{1}, \ldots, u_{n}\right)=\left(F_{1}, \ldots, F_{n}\right) \exp (\theta \log x)
$$

where $\theta$ is an $n \times n$ matrix with constant coefficients (and if you wish, rational eigenvalues). We are given that the wronskian of $\left(u_{1}, \ldots, u_{n}\right)$ is bounded in $D\left(0,1^{-}\right)$, we are to obtain estimates of type (3) for the $F_{i}$. This problem has been solved by Adolphson and Sperber for the case in which $\theta$ is nilpotent of order $n$ (i.e. one Jordan block). We do observe that without this restriction, the $u_{i}$ satisfy a linear differential equation (3.2) with meromorphic coefficients and if the differences of eigenvalues of $\theta$ are in $\mathbf{Z}_{p}$ then the effective bounds (3.6) are valid. We remind the reader that this information is useful for transfer to disks where (3.2) has no singularities but that this gives no direct information concerning the $F_{i}$.

We now give a quick review of the history of these questions. Conditions of type (3) appear explicitly [2, Theorem 3.2, p. 238] in connection with the study of solutions of certain first order partial differential equations. The basic principle involved the use of magnitude of eigenvalues of Frobenius to establish growth conditions. The same argument appears in a more general setting in Katz's article $[8, \S 3.1 .2]$ where he shows that horizontal elements of $F$-crystals must converge in a disk of radius unity, cf. [3, Theorem 6, p. 65], but it could have been used to obtain bounds of type (3) for these horizontal elements. The estimates obtained from these cohomological considerations are in general quite sharp as they involve values (in the logarithmic scale) of eigenvalues of Frobenius rather than the order of the differential equation which is in general larger. 
The subject was freed from considerations of $p$-adic cohomology in [4] where (with the improvement of [10, (4.25)]), (3) was demonstrated under the hypothesis that $u$ is in the span of $n$ functions analytic on $D\left(0,1^{-}\right)$which satisfy a linear differential equation with coefficients in $K(x)$. The extension to the case in which the coefficients are analytic elements was given in $[6,(4.2 .6)]$ the case in which the coefficients are algebraic functions was given in [5, Corollary 4.5] and the case in which we only demand that the coefficients be bounded was given in [11, Theorem 5.6].

We are indebted to E. Bombieri for bringing the question of effective estimates to our attention. The exposition has been improved by advice from S. Sperber and A. Adolphson concerning the removal of considerations of the generic disk from our work. Finally $\$ 7$ has been affected by conversations with N. Katz.

1. Meromorphic functions on a disk. Let $K$ be an algebraically closed field of characteristic zero complete under a nonarchimedean valuation of residue class $p$.

For each $a \in K$ and each positive real number $r$, let

$$
D\left(z, r^{-}\right)=\{x \in K|| x-a \mid<r\} \text {. }
$$

For $f \in K[[x-a]], f=\sum_{\nu=0}^{\infty} b_{\nu}(x-a)^{\nu}$, analytic on $D\left(a, r^{-}\right)$, let

$$
|f|_{a}(\rho)=\sup _{\nu}\left|b_{\nu}\right| \rho^{\nu} \quad \text { for } \rho<r .
$$

This is extended to functions $f$, meromorphic on $D\left(a, r^{-}\right)$by writing $f=g / h$ where both $g$ and $h$ are analytic on $D\left(a, r^{-}\right)$and letting

$$
|f|_{a}(\rho)=|g|_{a}(\rho) /|h|_{a}(\rho) \text {. }
$$

This ratio is known to be independent of the choice of $g$ and $h$. In particular $\rho \mapsto|f|_{a}(\rho)$ is continuous and if $f$ has no poles on the set $|x|=\rho$ then

$$
|f|_{a}(\rho)=\sup _{|x|=\rho}|f(x)| \text {. }
$$

If $f$ has no poles on the indicated set then $f$ may be represented on that set by a Laurent series

$$
f(x)=\sum_{\nu=-\infty}^{\infty} b_{\nu}(x-a)^{\nu}
$$

and we have

$$
|f|_{a}(\rho)=\sup _{\nu}\left|b_{\nu}\right| \rho^{\nu}
$$

This may be used to verify that if $f$ is meromorphic on $D\left(a, r^{-}\right)$then

$$
\left|\frac{1}{s !} f^{(s)}\right|_{a}(\rho)<\frac{1}{\rho^{s}}|f|_{a}(\rho) .
$$

In the following we shall use $Q_{0}$ to denote the ring of functions analytic on $D\left(0,1^{-}\right)$, i.e. of elements

$$
u=\sum b_{\nu} x^{\nu} \in K[[x]]
$$

such that

$$
\lim _{s \rightarrow \infty}\left|b_{s}\right| r^{s}=0 \quad \forall r<1
$$


Let $\mathbb{Q}_{0}^{\prime}$ denote the field of quotients of $\mathbb{Q}_{0}$.

We define the boundary seminorm \|\|$_{0}$ by setting

$$
\|u\|_{0}=\underset{r \rightarrow 1}{\lim \sup }|u|_{0}(r)
$$

for each $u \in \mathbb{Q}_{0}^{\prime}$. Note that $\|u\|_{0}=0$ does not imply $u=0$. Let $\mathscr{B}_{0}$ denote the ring of bounded analytic functions on $D\left(0,1^{-}\right)$and let $\mathscr{B}_{0}^{\prime}$ be the field of quotients of $\mathscr{B}_{0}$. Thus $\mathscr{B}_{0}^{\prime}$ is naturally imbedded in $\mathscr{Q}_{0}^{\prime}$ and the restriction of the boundary seminorm to $\mathscr{B}_{0}^{\prime}$ is a norm.

In particular

$$
\begin{gathered}
\|u v\|_{0}<\|u\|_{0}\|v\|_{0} \quad \text { if } u, v \in \mathbb{Q}_{0}^{\prime} \\
\|u v\|_{0}=\|u\|_{0}\|v\|_{0} \quad \text { if } u, v \in \mathscr{B}_{0}^{\prime} \\
\left\|\frac{1}{s !} D^{s} u / u\right\|_{0}<1
\end{gathered}
$$

for all $u \in Q_{0}^{\prime}$ while

$$
\left\|\frac{1}{s !} D^{s} u\right\|_{0}<\|u\|_{0}
$$

for all $u \in \mathscr{B}_{0}^{\prime}$.

(1.10) For use in $\S 8$ we observe that if $u=x^{\sigma} \xi$ with $\xi \in \mathbb{Q}_{0}^{\prime}, \sigma \in K$ then

$$
u^{\prime} / u=\sigma / x+\xi^{\prime} / \xi \in \mathbb{Q}_{0}^{\prime}
$$

and hence $u^{(s)} / u$, which lies in $Q\left[u^{\prime} / u,\left(u^{\prime} / u\right)^{\prime}, \ldots,\left(u^{\prime} / u\right)^{(s-1)}\right]$, is also in $\mathbb{Q}_{0}^{\prime}$. For $u=x^{\sigma}$ we have

$$
u^{(s)} / u s !=\left(\begin{array}{c}
\sigma \\
s
\end{array}\right) / x^{s}
$$

and hence (1.8) holds for all $s$ if and only if $\sigma \in \mathbf{Z}_{p}$. We conclude with the aid of Leibnitz's rule that estimate (1.8) is valid for $u=x^{\sigma} \xi$ if $\xi \in \mathbb{Q}_{0}^{\prime}$ and $\sigma \in \mathbf{Z}_{p}$.

(1.11) We now make some elementary observations concerning unbounded elements of $\mathbb{Q}_{0}$. These remarks will be used in $\$ 9$.

Let $g$ be an element of $\mathscr{Q}_{0}$ which is unbounded on $D\left(0,1^{-}\right)$. For each $r \in(0,1)$ let

$$
N_{g}(r)=\text { the number of zeros of } g \text { in } D\left(0, r^{-}\right) .
$$

It is well known that

$$
\frac{d \log |g|_{0}(r)}{d(\log r)}=N_{g}(r)
$$

whenever the right side is defined.

For each $s \in \mathbf{N}$, let

$$
\alpha_{s}=\underset{0<r<1}{\operatorname{Inf}}|g|_{0}(r) / r^{s}
$$

We choose $R_{s}$ maximal in $(0,1)$ such that

$$
N_{g}\left(R_{s}\right)<s .
$$

Clearly $R_{s} \rightarrow 1$ as $s \rightarrow \infty$. Furthermore equation (1.11.1) shows that

$$
\alpha_{s}=|g|_{0}\left(R_{s}\right) / R_{s}^{s} \text {. }
$$


We conclude that given $\varepsilon \in(0,1)$, we have

$$
\alpha_{s}=\operatorname{Inf}_{\varepsilon<r<1}|g|_{0}(r) / r^{s}
$$

for all $s>s_{0}(\varepsilon)$.

Let $m$ be a fixed positive integer and $u=\Sigma b_{s} x^{s}$ an element of $K[[x]]$ with asymptotic growth

$$
b_{s}=O\left(s^{m} \alpha_{s}\right) \text {. }
$$

Lemma. The series $u$ lies in $\mathbb{Q}_{0}$ and for $c \in D\left(0,1^{-}\right)$the translated series $u(c+x)$ satisfies the same asymptotic growth condition (1.11.6).

Proof. To show $u \in \mathbb{Q}_{0}$ we must show that for $r \in(0,1)$ we have

$$
\lim _{s \rightarrow \infty} s^{m} \alpha_{s} r^{s}=0 .
$$

We fix $\rho \in(r, 1)$ and restrict our attention to $s$ so large that $R_{s}>\rho$. It then follows from (1.11.4) that

$$
s^{m} \alpha_{s} r^{s}<|g|_{0}(\rho)(r / \rho)^{s} s^{m}
$$

The right side of (1.11.7.1) has limit zero as $s$ gives to infinity and this proves (1.11.7). We now write the translated function

$$
u(c+x)=\sum b_{s}^{\prime} x^{s}
$$

so that

$$
b_{s}^{\prime}=\sum_{\nu=0}^{\infty} b_{s+\nu} c^{\nu}\left(\begin{array}{c}
s+\nu \\
s
\end{array}\right)
$$

It follows from the definitions that

$$
\frac{|g|_{0}\left(R_{s+\nu}\right)}{R_{s+\nu}^{s+\nu}}<\frac{|g|_{0}\left(R_{s}\right)}{R_{s}^{s+\nu}}
$$

and hence

$$
\frac{\alpha_{s+v}}{\alpha_{s}}<\frac{1}{R_{s}^{\prime}}
$$

Thus

$$
\frac{\left|b_{s+\nu} c^{\nu}\right|}{\alpha_{s}}<(s+\nu)^{m}|c|^{\nu} \frac{\alpha_{s+\nu}}{\alpha_{s}}<(s+\nu)^{m}\left(\frac{|c|}{R_{s}}\right)^{\nu} .
$$

Choose $\rho \in(|c|, 1)$, choose $s_{0}$ such that

$$
R_{s_{0}}>\rho,\left(1+1 / s_{0}\right)^{m}<\rho /|c| .
$$

We conclude that for $s>s_{0}$, the extreme right-hand side of (1.11.9.2) is a function of $\nu \in \mathrm{N}$, maximal when $\nu=0$. We conclude that for $s>s_{0},\left|b_{s+\nu} c^{\nu}\right|$ is bounded from above by $\alpha_{s} \cdot s^{m}$ and so by (1.11.8.1) the same holds for $\left|b_{s}^{\prime}\right|$. This completes the proof of the lemma.

2. Formal integrals. Let $\mathfrak{\Re}$ be a differential ring of characteristic zero and with derivation $D$. Let $v_{1}, v_{2}, \ldots, v_{m}$, and $y_{1}, y_{2}, \ldots, y_{m}$ be elements of $\Re$, satisfying 
the relations

$$
y_{m}=1, \quad D y_{j}=v_{j+1} y_{j+1}, \quad 1<j<m-1 .
$$

(Putting $y_{j}=1$ for $j>m ; v_{j}=0$ for $j>m+1$, we note that (2.1) then holds for all $j \in \mathbf{N}^{*}$.) Informally we may write

$$
\begin{gathered}
y_{m-1}=D^{-1} v_{m}, \\
y_{m-2}=D^{-1}\left(v_{m-1} D^{-1} v_{m}\right) \\
\therefore \cdots \cdots \cdots \cdots \\
y_{1}=D^{-1}\left(v_{2}\left(D^{-1}\left(v_{3} \cdots D^{-1} v_{m}\right)\right)\right) .
\end{gathered}
$$

The parentheses will be dropped when no confusion can occur. For $1<k<m$, $s \in \mathbf{N}$ we define (with $v^{\left(\lambda_{1}\right)}=D^{\lambda_{1}} v$ )

$$
\begin{aligned}
B_{s, k}\left(v_{1}, \ldots, v_{k}\right)= & \sum \frac{v_{1}^{\left(\lambda_{1}\right)}}{\lambda_{1} !} \frac{v_{2}^{\left(\lambda_{2}\right)}}{\lambda_{2} !} \cdots \frac{v_{k}^{\left(\lambda_{k}\right)}}{\lambda_{k} !} \\
& \cdot \frac{1}{\left(1+\lambda_{k}\right)\left(2+\lambda_{k}+\lambda_{k-1}\right)\left(k-1+\lambda_{k}+\cdots+\lambda_{2}\right)} .
\end{aligned}
$$

The sum being over all $\lambda_{1}, \ldots, \lambda_{k}$ in $\mathbf{N}$ such that

$$
\lambda_{1}+\lambda_{2}+\cdots+\lambda_{k}=s-(k-1)
$$

so in particular

$$
\begin{aligned}
B_{s, k} & =0 \text { for } k>s+1, \\
B_{s, k}\left(v_{1}, \ldots, v_{k}\right) & =v_{1} v_{2} \cdots v_{s+1} \cdot \frac{1}{s !} \text { for } k=s+1, \text { and } \\
B_{0, k} & =0 \text { for } k>1, \\
B_{0,1} & =v_{1} .
\end{aligned}
$$

LEMMA. For each integer $s \in \mathbf{N}$,

$$
\begin{aligned}
& \frac{1}{s !} D^{s}\left(v_{1} y_{1}\right)=B_{s, 1}\left(v_{1}\right) y_{1}+B_{s, 2}\left(v_{1}, v_{2}\right) y_{2}+\cdots+B_{s, m}\left(v_{1}, \ldots, v_{m}\right) \\
&\left(=\sum_{j=1}^{\infty} B_{s, j}\left(v_{1}, \ldots, v_{i}\right) y_{i} \text { if we set } v_{j}=0 \text { for } j>m\right) .
\end{aligned}
$$

Proof. For $m=1$, the assertion is that

$$
\frac{1}{s !} D^{s}\left(v_{1}\right)=B_{s, 1}\left(v_{1}\right)
$$

which is clear. We use induction on $m$. By Leibnitz's rule

$$
\begin{aligned}
\frac{1}{s !} D^{s}\left(v_{1} y_{1}\right) & =\sum_{j+t=s} \frac{1}{j !} v^{(j)} \frac{1}{t !} y_{1}^{(t)} \\
& =\frac{1}{s !} v^{(s)} y_{1}+\sum_{\substack{t>1 \\
t+\lambda_{1}=s}} \frac{1}{t !}\left(y_{1}^{\prime}\right)^{t-1} \frac{1}{\lambda_{1} !} v^{\left(\lambda_{1}\right)}
\end{aligned}
$$


so

$$
\frac{1}{s !} D^{s}\left(v_{1} y_{1}\right)-B_{s, 1}\left(v_{1}\right) y_{1}=\sum_{\substack{t+\lambda_{1}=s \\ t>1}} \frac{1}{t !}\left(v_{2} y_{2}\right)^{(t-1)} \frac{1}{\lambda_{1} !} v_{1}^{\left(\lambda_{1}\right)}
$$

So by the induction hypothesis applied to $v_{2}, v_{3}, \ldots, v_{m}$, we have

$$
\begin{aligned}
\frac{1}{(t-1) !} D^{t-1}\left(v_{2} y_{2}\right)= & B_{t-1,1}\left(v_{2}\right) y_{2}+B_{t-1,2}\left(v_{2}, v_{3}\right) y_{3}+\cdots \\
& +B_{t-1, m-1}\left(v_{2}, \ldots, v_{m}\right) y_{m} .
\end{aligned}
$$

Substitution in the right side of (2.4) for $t>1$ gives the coefficient of $y_{l}$ to be

$$
\sum_{\substack{t+\lambda_{1}=s \\ t>1}} \frac{1}{t} B_{t-1, l-1}\left(v_{2}, \ldots, v_{l}\right) \frac{1}{\lambda_{1} !} v_{1}^{\left(\lambda_{1}\right)}
$$

and it follows from the definition that this sum coincides with $B_{s, l}\left(v_{1}, \ldots, v_{l}\right)$ for $l>2$. This completes the proof of the lemma.

For later use we define

$$
A_{s, k}\left(v_{1}, \ldots, v_{k}\right)=\frac{1}{v_{1} v_{2} \cdots v_{k}} B_{s, k}\left(v_{1}, \ldots, v_{k}\right)
$$

Proposition. For $v_{1}, \ldots, v_{m} \in \mathbb{Q}_{0}^{\prime}$, we have (see definition (3.6.1) below)

$$
\left\|A_{s, k}\left(v_{1}, \ldots, v_{k}\right)\right\|_{0}<\{s, k-1\}_{p} \text {. }
$$

Proof. This follows from equations (2.2) and (1.8).

Corollary.

$$
\left\|s ! A_{s, k}\left(v_{1}, \ldots, v_{k}\right)\right\|_{0}<1
$$

3. Effective bounds for boundary seminorms. Let $u_{1}, \ldots, u_{n}$ be elements of $Q_{0}^{\prime}$ with nontrivial wronskian. Let $\Delta$ be the $n \times n$ diagonal matrix whose general coefficient is given by

$$
\Delta_{\alpha, \alpha}=(\alpha-1) !
$$

Let $W$ be the modified wronskian matrix

$$
W=W\left(u_{1}, \ldots, u_{n}\right)=\Delta^{-1}\left(\begin{array}{cccc}
u_{1} & u_{2} & \cdots & u_{n} \\
u_{1}^{\prime} & u_{2}^{\prime} & \cdots & u_{n}^{\prime} \\
u_{1}^{(n-1)} & . & \cdots & u_{n}^{(n-1)}
\end{array}\right)
$$

The $n$ functions $u, \ldots, u_{n}$ span an $n$-dimensional $K$ space which is the kernel in $\mathbb{Q}_{0}^{\prime}$ of a uniquely determined differential operator

$$
L=D^{n}+P_{n-1} D^{n-1}+\cdots+P_{0} \in \mathbb{Q}_{0}^{\prime}[D] .
$$

For each integer $s>0$ we write

$$
\frac{1}{s !} D^{s} \equiv \sum_{j=0}^{n-1} G_{s, j} D^{j} \bmod \mathbb{Q}_{0}^{\prime}[D] L
$$


and this uniquely determines each $G_{s, j} \in \mathbb{Q}_{0}^{\prime}$. It will be convenient to write $G_{s, j}=H_{s, j} / j$ ! for $s \in \mathrm{N}, 0<j<n-1$, so that

$$
\frac{1}{s !} D^{s} \equiv \sum_{j=0}^{n-1} H_{s, j} \frac{1}{j !} D^{j} \bmod \mathbb{Q}_{0}^{\prime}[D] L .
$$

We simplify notation by letting $U, H, G$, be $\infty \times n$ matrices whose $(s+1)$ th rows are

$$
\frac{1}{s !} D^{s}\left(u_{1}, \ldots, u_{n}\right)
$$

resp.:

$$
H_{s, 0}, \ldots, H_{s, n-1}
$$

resp.:

$$
G_{s, 0}, \ldots, G_{s, n-1}
$$

Hence

$$
\begin{gathered}
G \Delta=H, \\
U=H W
\end{gathered}
$$

TheOREM. For all $s \in \mathbf{N}, 0<j<n-1$,

$$
\left\|G_{s, j}\right\|_{0}<\{s, n-1\}_{p} \text {. }
$$

3.6.1 Definition. $\{s, k\}_{p}$ is the reciprocal of Inf $\left|\lambda_{1} \cdots \lambda_{k}\right|_{p}$, the infimum being over all sets of $k$ distinct natural numbers bounded by $s$, i.e., $1<\lambda_{1}<\lambda_{2}$ $<\cdots<\lambda_{k}<s$.

Proof. We use the classical construction [7, p. 264] giving a decomposition of $L$ into linear factors (in $\left.\mathbb{Q}_{0}^{\prime}[D]\right)$. We choose $v_{1}, \ldots, v_{n} \in \mathbb{Q}_{0}^{\prime}$ such that

$$
\begin{gathered}
u_{1}=v_{1}, \\
u_{2}=v_{1} D^{-1} v_{2}, \\
\cdots \cdots \cdots \\
u_{n}=v_{1} D^{-1} v_{2} \cdots D^{-1} v_{n} .
\end{gathered}
$$

3.7.1 REMARK.

$$
L=L_{n} \circ L_{n-1} \circ \cdots \circ L_{1}
$$

where

$$
L_{j}=v_{1} \cdots v_{j} \circ D \circ \frac{1}{v_{1} \cdots v_{j}} .
$$

We now continue the proof of (3.6). We use Lemma 2.3 to obtain

$$
\begin{aligned}
\frac{1}{s !} D^{s} u_{k} & =\frac{1}{s !} D^{s}\left(v_{1} D^{-1} v_{2} \cdots D^{-1} v_{k}\right) \\
& =\sum_{i=1}^{k} B_{s, i}\left(v_{1}, \ldots, v_{i}\right) D^{-1} v_{i+1} D^{-1} v_{i+2} \cdots D^{-1} v_{k}
\end{aligned}
$$

and so deduce

$$
\boldsymbol{U}=\boldsymbol{B} \boldsymbol{J}
$$


where $B$ is $\infty \times n$ matrix whose $(s+1)$ th row is $\left(B_{s, 1}\left(v_{1}\right), \ldots, B_{s, n}\left(v_{1}, \ldots, v_{n}\right)\right)$ and $J$ is the upper triangular $n \times n$ matrix whose coefficients are given by

$$
\begin{aligned}
J_{\alpha, \beta} & =0 & & \text { if } \beta-\alpha<0, \\
& =1 & & \text { if } \beta-\alpha=0, \\
& =D^{-1} v_{\alpha+1} \cdots D^{-1} v_{\beta} & & \text { if } \beta-\alpha>1,
\end{aligned}
$$

i.e.

$$
J=\left(\begin{array}{cccc}
1 & D^{-1} v_{2} & D^{-1} v_{2} D^{-1} v_{3} & \cdots \\
0 & 1 & D^{-1} v_{3} & \\
\cdot & \cdots & \cdots & \cdots
\end{array}\right] .
$$

We now write $W$ in terms of (3.8). Indeed $W$ is a truncation $U_{n}$ consisting of the first $n$ rows of $U$ and so

$$
W=U_{n}=B_{n} J
$$

where $B_{n}$ denotes the first $n$ rows of $B$.

3.10.1 REMARK. For latter use we note that since $J$ is upper triangular and since $B_{n}$ is lower triangular (2.2.1), we may calculate the conventional wronskian determinant $\mathfrak{m}\left(u_{1}, \ldots, u_{n}\right)$ of $u_{1}, \ldots, u_{n}$ to be $v_{1}^{n} v_{2}^{n-1} \ldots v_{n}$. Since the $v_{1}, \ldots, v_{s}$ are determined by $u_{1}, \ldots, u_{s}$, we may truncate (3.7) and so we verify the wellknown formula [7a, p. 256] (Ince p. 120).

$$
v_{s+1}=\mathfrak{w}\left(u_{1}, \ldots, u_{s+1}\right) \mathfrak{w}\left(u_{1}, \ldots, u_{s-1}\right) / \mathfrak{m}\left(u_{1}, \ldots, u_{s}\right)^{2} .
$$

We now resume the proof of (3.6). By comparing (3.5) and (3.8) and applying (3.10),

$$
B J=U=H W=H B_{n} J .
$$

Since $J$ is invertible, we conclude that

$$
B=H B_{n} \text {. }
$$

Multiplying both sides on the right by the $n \times n$ diagonal matrix

$$
\left(\begin{array}{cccc}
v_{1} & & & \\
& v_{1} v_{2} & & \\
& & \ddots & \\
& & & v_{1} \cdots v_{n}
\end{array}\right)^{-1}
$$

we obtain

$$
A=H A_{n}
$$

where $A$ is the $\infty \times n$ matrix whose $(s+1)$ th row is $\left(A_{s, 1}\left(v_{1}\right), \ldots, A_{s, n}\left(v_{1}, \ldots, v_{n}\right)\right)$ and $A_{n}$ is the $n \times n$ matrix consisting of the first $n$ rows of $A$. We now apply (3.4) to rewrite (3.11') as

$$
A=G \Gamma
$$

where $\Gamma=\Delta A_{n}$. The general term of $\Gamma$ is

$$
\Gamma_{\alpha, \beta}=(\alpha-1) ! A_{\alpha-1, \beta}\left(v_{1}, \ldots, v_{\beta}\right), \quad 1<\alpha, \beta<n .
$$


It follows from (2.2.1) and from (2.9) that

$$
\begin{aligned}
\Gamma_{\alpha, \beta}=0 & \text { for } \beta>\alpha, \\
\Gamma_{\alpha, \alpha}=1 & \text { for } 1 \leqslant \alpha<n, \\
\left\|\Gamma_{\alpha, \beta}\right\|_{0}<1 & \text { for } 1<\alpha, \beta<n .
\end{aligned}
$$

These three properties must hold for $\Gamma^{-1}$ and since (3.11.1) implies

$$
A \Gamma^{-1}=G
$$

we see that

$$
\left\|G_{s, j}\right\|_{0}<\operatorname{Sup}_{1<k<n}\left\|A_{s, k}\left(v_{1}, \ldots, v_{k}\right)\right\|
$$

(in fact we may take $\operatorname{Sup}_{j<k<n}$ ). The theorem now follows from (2.8).

Corollary.

$$
\left\|P_{j}\right\|_{0}<1, \quad j=0,1, \ldots, n-1 \text {. }
$$

Proof. This follows from (3.2) which shows that $P_{j}=-n ! G_{s, j}$ and hence the assertion follows from the theorem. However this well-known relation may also be deduced from (3.7), using induction on $n$.

4. Analytic functions with bounded wronskian. Let $u_{1}, u_{2}, \ldots, u_{n}$ be elements of $Q_{0}$ (i.e. analytic on $D\left(0,1^{-}\right)$rather than merely meromorphic). We use the same notation as in $\$ 3$ but we make the further hypothesis that not only is det $W$ nontrivial but in addition (letting $w=\operatorname{det} W$ )

$$
\|w\|_{0}=\|\operatorname{det} W\|_{0}<\infty
$$

(Since $\operatorname{det} W \in \mathbb{Q}_{0}$, $\|\operatorname{det} W\|_{0} \neq 0$.)

4.2 TheOREM. If $u_{1}, \ldots, u_{n} \in \mathbb{Q}_{0}$ and if $w=\operatorname{det} W$, the (modified) wronskian, is bounded as function on $D\left(0,1^{-}\right)$then each $u_{j}$ may be written as a power series

$$
\sum a_{s} x^{s}
$$

with

$$
\left|a_{s}\right|=O\left(s^{n-1}\right)
$$

Remark. This result is a very slight improvement upon [12, Theorem 5.6]. As noted previously the exposition is totally different.

Proof. Letting Adj $W$ denote the transpose of the matrix of cofactors of $W$, we deduce from equation (3.5) that

$$
U \text { Adj } W=H \cdot \operatorname{det} W \text {. }
$$

The matrix coefficients on the left side lie in $\mathbb{Q}_{0}$ and hence using (3.4) we conclude that $G_{s, j}$ det $W$ is analytic on $D\left(0,1^{-}\right)$. By the maximum principle for $x \in$ $D\left(0,1^{-}\right)$,

$$
\left|\left(G_{s, j} w\right)(x)\right|<\left\|G_{s, j} w\right\|_{0}<\left\|G_{s, j}\right\|_{0}\|w\|_{0}
$$

By (4.1), (3.6), this gives

$$
\left|\left(G_{s, j} w\right)(x)\right|<\|w\|_{0}\{s, n-1\}_{p} .
$$


Let $c$ be chosen in $D\left(0,1^{-}\right)$such that

$$
w(c) \neq 0
$$

We deduce

$$
\left|G_{s, j}(c)\right|<k\{s, n-1\}_{p},
$$

where $k=\|w\|_{0} /|w(c)|$. The $K$-span of $u_{1}, \ldots, u_{n}$ has $\tilde{u}_{1}, \ldots, \tilde{u}_{n}$ as basis where

$$
\tilde{u}_{j}(x)=\sum_{s=0}^{\infty} G_{s, j}(c)(x-c)^{s}, \quad(j=0,1, \ldots, n-1),
$$

is the unique element of the kernel of $L$ which satisfies the initial conditions

$$
\tilde{u}_{j}^{(i)}(c)=\delta_{i, j}
$$

It is trivial that $\tilde{u}_{j}$ satisfies (4.2) as power series in $(x-c)$ and so it is enough to check that this condition is not affected by the change in variable $x \rightarrow(x-c)$. This follows easily from $|c|<1$.

4.3 THEOREM. If $u_{1}, \ldots, u_{n}$ are elements of $\mathbb{Q}_{0}$ whose wronskian $w$ is never zero on $D\left(0,1^{-}\right)$then for each element $u$ in the $K$-span of $u_{1}, \ldots, u_{n}$ we have the effective bounds, $c f$. (3.6.1):

$$
\begin{gathered}
u=\sum_{s=0}^{\infty} a_{s} x^{s}, \\
\left|a_{s}\right| \leqslant\{s, n-1\}_{p} \operatorname{Sup}_{0<k<n-1}\left|a_{k}\right| .
\end{gathered}
$$

Proof. Since $w$ is never zero on $D\left(0,1^{-}\right)$, we know that $w$ is surely bounded and in fact

$$
|w(0)|=\|w\|_{0} .
$$

Thus letting $c=0$ in the proof of Theorem 4.2, we see that $k=1$ in equation (4.2.5) and since equation (4.3.1) implies that

$$
u=\sum_{j=0}^{n-1} a_{j} \tilde{u}_{j},
$$

it is clear that (4.3.2) is a consequence of (4.2.5).

REMARK. The hypothesis of Theorem 4.2 implies by (3.15) that each $P_{j}$ lies in $\mathscr{B}_{0}^{\prime}$ and that $w P_{j}$ lies in $\mathscr{B}_{0}$.

Clearly under the hypothesis of 4.3 , each $P_{j}$ lies in $\mathscr{B}_{0}$ and is bounded by 1 .

5. Apparent singularities. The object of this paragraph is to obtain an effective form for Theorem 4.2 without insisting upon the analyticity of the reciprocal of the wronskian.

Let $K$ be as in $\S 1$ and let $K_{0}$ be an arbitrary field of characteristic zero with a chosen imbedding of $K_{0}$ in $K$. (Thus $K_{0}$ need be neither complete nor algebraically closed, but a valuation, $\mathfrak{p}$, of $K_{0}$ has been chosen.)

Let $u_{1}, \ldots, u_{n}$ be elements of $K_{0}[[x]]$ which converge in $D\left(0, r_{p}^{-}\right)$. The wronskian $w=w\left(u_{1}, \ldots, u_{n}\right)$ is independent of the valuation $\mathfrak{p}$. As before we assume that $w$ is not identically zero. We assume that $|w|_{0}\left(r_{p}\right)$ is finite. In the notation of (3.3), 
equation (3.6) may now be written

$$
\left|G_{s, j}\right|_{0}\left(r_{\mathfrak{p}}\right)<\{s, n-1\}_{p} r_{\mathfrak{p}}^{-s+j}
$$

It follows from the proof of Theorem 4.2 that each $u_{j}$ is of the form $\Sigma a_{s} x^{s}$ with

$$
\left|a_{s}\right|=O\left(s^{n-1} / r_{\mathfrak{p}}^{s}\right)
$$

We now make this estimate effective.

LEMMA. If $w(0) \neq 0$ then $\left(5.1^{\prime}\right)$ may be replaced by

$$
\left|a_{s}\right|<\frac{|w|_{0}}{|w(0)|}\left(r_{\mathfrak{p}}\right) r_{\mathfrak{p}}^{-s}\{s, n-1\}_{p} \sup _{0<k<n-1}\left\{\left|a_{k}\right| r_{\mathfrak{p}}^{k}\right\}
$$

Proof. In the proof of Theorem 4.2 we may take $c=0$. The estimate for $\left|G_{s, j}(0)\right|$ is then given by (4.2.5) modified so as to read

$$
\left|G_{s, j}(0)\right|<\{s, n-1\}_{p} r_{\mathfrak{p}}^{-s+j}|w|_{0}\left(r_{\mathfrak{p}}\right) /|w(0)| \text {. }
$$

The lemma then follows from the basis $\left\{\tilde{u}_{j}\right\}_{0<j<n-1}$ (given by (4.2.6) with $c=0$ ) of the $K_{0}$-span of $u_{1}, \ldots, u_{n}$.

With the object of being able to analyze the case in which $w(0)=0$ we consider the functions in two variables

$$
u_{j}(x, z)=\sum_{s=0}^{\infty} w(x) G_{s, j}(x) z^{s}, \quad 0<j<n-1 .
$$

Letting $R$ be the ring of elements of $K_{0}[[x]]$ which are bounded on $D\left(0, r_{\mathfrak{p}}^{-}\right)$we conclude

\subsection{Proposition.}

(5.4.1) For $j=0,1, \ldots, n-1, u_{j}(x, z)$ is an element of $R[[z]]$ which is analytic as $a$ function of $x$ and $z$ on the product of $D\left(0, r_{p}^{-}\right)$with itself.

(5.4.2) For each $x_{0} \in D\left(0, r_{p}^{-}\right)$, the functions $x \mapsto u_{j}\left(x_{0}, x-x_{0}\right)$ lie in the kernel of $L$ (equation (3.2)) and hence in the $K$-span of $u_{1}, \ldots, u_{n}$.

(5.4.3) If $w\left(x_{0}\right) \neq 0, x_{0} \in D\left(0, r_{p}^{-}\right)$then the functions of (5.4.2) form a basis of the $K$-span of $u_{1}, \ldots, u_{n}$.

(5.4.4) There exists a matrix $M \in G L\left(n, K_{0}\left[x, x^{-1}\right]\right)$ with coefficients in $K\left[x^{-1}\right]$ such that the $\infty \times n$ matrix $w G M$ (recall $G$ is defined as in equation (3.4)) has coefficients in $R$ and has reduction modulo $x$ which is in Hermite normal form.

(5.4.5) Writing $f=w G M$, the reduction of $f$ modulo $x R$ may be represented by $a$ matrix $\bar{f}=f(0)$ with coefficients in $K_{0}$ having the property that there exist $n$ rows $t_{1}<t_{2}<\cdots<t_{n}$ with the property that

$$
\bar{f}_{i, j}=\delta_{i, j}, \quad 0<i, j<n-1
$$

(we index the rows by $\mathbf{N}$ and the columns by $\{0,1, \ldots, n-1\}$ ). Also

$$
\bar{f}_{s, j}=0 \quad \text { for } s<t_{j} \text {. }
$$

Proof. Statements (5.4.1)-(5.4.3) follow from the definitions. Assertions (5.4.4) and (5.4.5) are well known. We recall that $M$ is constructed so as to perform the 
following operations on the columns of $G$ :

$(\alpha)$ columns may be interchanged,

( $\beta$ ) columns with coefficients in $x^{b} R(b>0)$ may be multiplied by $x^{-b}$,

$(\gamma)$ we may add to one column a $K_{0}$ multiple of the other.

This completes our discussion of the proposition.

Let us use $R^{\prime}$ to denote the quotient field of $R$, the ring of bounded analytic functions on $D\left(0, r_{\mathfrak{p}}^{-}\right)$(which are represented by elements of $\left.K_{0}[[x]]\right)$. The $\mid l_{0}\left(r_{\mathfrak{p}}\right)$ norm on $R^{\prime}$ may be extended to a norm on $R^{\prime n}$, the space of $n$-tuples. The matrix $M$ of the above proposition operates on $R^{\prime n}$. This assigns a norm to the matrix $M$ which we denote by $\|M\|\left(\mathfrak{p}, r_{p}\right)$.

\subsection{Proposition.}

$$
\|M\|\left(\mathfrak{p}, r_{\mathfrak{p}}\right) \leqslant C_{\mathfrak{p}}\left(\operatorname{Inf}\left(1, r_{\mathfrak{p}}\right)\right)^{-\operatorname{deg} M}
$$

where $\operatorname{deg} M$ refers to degree as polynomial in $x^{-1}$ with coefficients which are $n \times n$ matrices in $K_{0}$, and where $C_{\mathfrak{p}}$ is maximum in the $\mathfrak{p}$ valuation of the elements of $K_{0}$ which appear as coefficients in $M$. In particular if $K_{0}$ is an algebraic number field then $C_{\mathfrak{p}}=1$ for almost all $\mathfrak{p}$.

Proof. This follows from the definitions.

5.6 THEOREM. Let $u_{1}, \ldots, u_{n}$ be elements of $K_{0}[[x]]$ which converge in $D\left(0, r_{p}^{-}\right)$ and with wronskian $w$ bounded in $D\left(0, r_{p}^{-}\right)$. Let $M$ be the matrix and $T=$ $\left\{t_{1}, \ldots, t_{n}\right\}$ be the set of $n$ distinct integers defined in Proposition (5.4). For $u$ in the $K_{0}$-span of $u_{1}, \ldots, u_{n}$ we have $u=\sum a_{s} x^{s}$ with

$$
\left|a_{s}\right| \leqslant\|M\|\left(\mathfrak{p}, r_{\mathfrak{p}}\right)|w|_{0}\left(r_{\mathfrak{p}}\right)\{s, n-1\}_{p} \sup _{t \in T}\left\{\left|a_{t}\right| r_{\mathfrak{p}}^{t}\right\}
$$

REMARK. The matrix $M$ depends on the normalization of $w$ but the product $|w|_{0}\left(r_{\mathfrak{p}}\right)\|M\|\left(\mathfrak{p}, r_{\mathfrak{p}}\right)$ does not.

Proof. It follows from the proposition that

$$
\xi_{j}(x)=\sum_{s=0}^{\infty} f_{s, j}(0) x^{s}, \quad j=0,1, \ldots, n-1,
$$

is a basis of the $K_{0}$-span of $u_{1}, \ldots, u_{n}$ and that the coefficient of $x^{4}$ in $\xi_{j}$ is $\delta_{i, j}$. Thus it is enough to verify the theorem for the $\xi_{j}$. We use the fact that

$$
f=w G M
$$

and so the sth row of $f$ has $R^{\prime n}$ norm bounded by the product of the corresponding norm of the $s$ th row of $w G$ with the corresponding transformation norm of the matrix $M$. These norms are given by (5.1) and (5.5). Finally we observe that $f_{s, j}$ may be specialized at $x=0$ for each $s, j$ and it is clear that $\left|f_{s, j}(0)\right|$, the $\mathfrak{p}$-valuation of this specialization, is bounded by $\left|f_{s, j}\right|_{0}\left(r_{p}\right)$. This completes the proof of the theorem.

6. Matrices of linear differential operators. The object of this section is to give an exposition of the fact that systems of linear ordinary differential equations are equivalent to a single equation. Our treatment should be viewed as a more 
complete development of the ideal theoretic method explained by Poole [9, p. 40]. (We are indebted to G. Hunt for drawing our attention to N. Jacobson, Pseudo-linear transformations, Ann. of Math. 38 (1937), his Theorems 3 and 4 contain our Lemmas 6.1, 6.2.) The question has also been treated by Deligne [1, p. 42].

Let $F$ be a field of characteristic zero, $D$ a nontrivial derivation of $F$ and $D$ the ring $F[D]$ of polynomials in $D$ with coefficients in $F$. As is well known, $D$ is a two-sided Euclidean ring and hence right ideals and left ideals are principal. Note that for $z \in F$, we have

$$
D \circ z-z D=z^{\prime}=D z
$$

6.1 LeMMA. $\mathfrak{D}$ is simple, i.e. each two-sided ideal is trivial.

Proof. Let $I$ be such an ideal and let

$$
\xi=a_{s} D^{s}+a_{s-1} D^{s-1}+\cdots+a_{0}, \quad a_{s} \neq 0,
$$

be an element of $I$ of minimal degree. Let $z$ be an arbitrary element of $F$. Then

$$
\xi \circ z-z \circ \xi=s a_{s} z^{\prime} D^{s-1}+\text { terms of lower degree. }
$$

The left-hand side shows that this element lies in $I$ but the hypothesis of minimality implies that it must be the zero element. Thus

$$
s a_{s} D z=0
$$

for all $z \in F$, but $D$ is not trivial, hence $s=0$ as asserted.

6.2 Lemma. If $A$ is an $n \times n$ matrix with coefficients in $D$ then there exist $\mathbf{G}, \mathbf{H}$ invertible $n \times n$ matrices with coefficients in $\bigcirc$ such that

$$
\mathbf{G} \boldsymbol{A} \mathbf{H}=\boldsymbol{J}
$$

where $J$ is a diagonal matrix whose coefficients are either zero or 1 with at most one exception.

Proof. By the theory of elementary divisors we may assume a diagonalization of $A$ with diagonal entries $\left(\alpha_{1}, \ldots, \alpha_{n}\right)$ the degree of $\alpha_{1}$ being minimal among all possible diagonalizations. We need only consider the case $n=2$.

By an elementary transformation,

$$
\left(\begin{array}{cc}
\alpha_{1} & 0 \\
0 & \alpha_{2}
\end{array}\right)
$$

is equivalent to

$$
\left(\begin{array}{cc}
\alpha_{1} & \lambda \alpha_{2} \\
0 & \alpha_{2}
\end{array}\right)
$$

for $\lambda \in \mathscr{D}$ and hence

$$
\lambda \alpha_{2}=\alpha_{1} \mu
$$

for some $\mu \in \mathfrak{D}$ as otherwise we could reduce to a diagonal form with an entry of lower degree. Since this holds for all $\lambda \in \mathfrak{D}$ we have

$$
\mathfrak{D} \alpha_{2} \subset \alpha_{1} \mathfrak{D}
$$


and hence

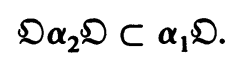

By the same argument

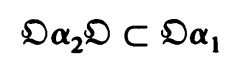

and so

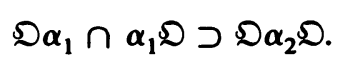

More generally

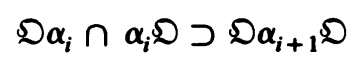

for $i=1,2, \ldots, n-1$. The left side of this last relation is closed under addition and hence we may replace the right side by $\mathbb{Q}_{i+1}$ the closure of the right side under addition.

But $\mathbb{Q}_{i+1}$ is a two-sided ideal and hence is either 0 or $\mathfrak{D}$. If 0 then $\alpha_{i+1}=0$ and $\alpha_{j}=0$ for all $j>i+1$. If $\alpha_{i+1} \neq 0$ then

$$
\mathfrak{D} \alpha_{i} \cap \alpha_{i} \mathfrak{D}=\mathfrak{D}
$$

and so $\alpha_{i}$ is a unit in $\mathfrak{D}$. If now $s-1$ is maximal such that $\alpha_{s-1}$ is a unit then $\alpha_{s}$ is not a unit and hence $\alpha_{s+1}=0$. Thus we may set $\alpha_{i}=1$ for $1<i<s-1, \alpha_{i}=0$ for $s+1<i<n$. We only know that $\alpha_{s}$ is not a unit but it may of course be zero.

6.3 LEMMA. Let $F$ be a subfield of the field $\Omega$ of germs of meromorphic functions at $x=0$ on a field $K$ as in $\$ 1$. Let $\Lambda$ be an $n \times n$ matrix with coefficients in $F$ and let $\mathfrak{D}=F[D]$ as above. Let $A=\Lambda-D$. By Lemma 6.2

$$
\Lambda-D=\mathbf{G}^{-1} J \mathbf{H}^{-1}
$$

where $\mathbf{G}, \mathbf{H}$ lie in $G L(n, \mathfrak{D})$ and $J$ is a diagonal matrix with coefficients in $\mathfrak{D}$. We now have

$$
J_{i i}=1, \quad i<n, J_{n n}=\text { polynomial in } D \text { of degree } n .
$$

Proof. This follows from the fact that for $x$ close to zero, $A$ operates on the $n$-fold sum of the $K$-space of germs analytic at $x$ with kernel which is a $K$-space of dimension $n$. This completes the proof.

6.4 REMARK. If

$$
y=\left(\begin{array}{c}
y_{1} \\
\vdots \\
y_{n}
\end{array}\right)
$$

is in the kernel of $\Lambda-D$ then

$$
\mathbf{H}^{-1} y=\left(\begin{array}{c}
0 \\
0 \\
\vdots \\
0 \\
u
\end{array}\right)
$$


where $J_{n n} u=0$. Thus if $\mathfrak{Y}$ is a solution matrix of $A$ then $\mathbf{H}^{-1} \mathfrak{Y}$ is an $n \times n$ matrix each of whose rows is zero except for the last row which is a set of $n$ independent solutions of $J_{n n}$.

7. Systems of linear differential equations. The preceding section may be used to deduce from our analysis of single equations (3.2) some information concerning systems of linear differential equations. We do not enter into the details. We do wish to point out an error to be avoided in these considerations.

It is natural to try to approach this question in the generality of $\S 4$, replacing the wronskian matrix $W$ by an $n \times n$ matrix $\mathfrak{Y}$ with coefficients in $\mathbb{Q}_{0}$ and replacing the condition that the wronskian determinant, $w$, is not identically zero and is bounded on $D\left(0,1^{-}\right)$, by the condition that det $\mathfrak{Y}$ is not identically zero and is bounded (or, say, never zero) on $D\left(0,1^{-}\right)$. We may view $\mathfrak{Y}$ as the solution matrix of $D-\Lambda$ where $\Lambda=\mathfrak{Y}^{\prime} \mathfrak{Y}^{-1}$ is a matrix with coefficients in $\mathcal{Q}_{0}^{\prime}$. Letting $F$ be $\mathscr{Q}_{0}^{\prime}$ in (6.3) we find that $D-\Lambda$ is equivalent to $J_{n n}$, an $n$th order linear operator with coefficients in $Q_{0}^{\prime}$. Now $J_{n n}$, does annihilate $n$ independent elements of $Q_{0}^{\prime}$ and these are the coefficients of the $n$th row of $\mathbf{H}^{-1} \mathfrak{Y}$. The estimates of $\$ 3$ are thus valid for $J_{n n}$. However while the coefficients of $\mathfrak{Y}$ lie in $\mathscr{C}_{0}$, we do not know that the solutions of $J_{n n}$ lie in this ring, we do not know a priori that the wronskian of $J_{n n}$ lies in the field $\mathscr{B}_{0}^{\prime}$. Even if we did know that $J_{n n}$ satisfied the hypotheses of Theorem 4.2, this would not help as the transformation of $\$ 6.4$ between $\mathfrak{V}$ and solutions of $J_{n n}$ is defined over $\mathscr{Q}_{0}^{\prime}$ (rather than over $\mathfrak{B}_{0}^{\prime}$ ) so no estimates may be deduced.

As an example, let $\mathfrak{Y}=\left(\begin{array}{ccc}1+u & u \\ 1 & 1\end{array}\right)$ with $u \in \mathbb{Q}_{0}$. Clearly no growth conditions may be deduced for $u$. These objections would be removed if we imposed upon $\mathfrak{V}$ the condition that $\mathfrak{Y}^{\prime} \mathfrak{Y}^{-1}$ have coefficients in $\mathscr{B}_{0}^{\prime}$.

7.1 Note added in proof. E. Bombieri has provided a definitive solution to this question which avoids $\$ 6$ and the calculation of an equivalent $n$th order linear differential equation.

Let $Y \in G L\left(n, Q_{0}^{\prime}\right)$ and for each integer $s \geqslant 0$ define a matrix $A_{s}$ by

$$
A_{s} Y=s !^{-1} D^{s} Y \text {. }
$$

Then

$$
\left\|A_{s}\right\|_{0} \leqslant\{s, n-1\} \operatorname{Sup}_{i<n-1}\left\|A_{i}\right\|_{0}
$$

The proof can be briefly indicated. Let $u=\left(u_{1}, \ldots, u_{n}\right)$ be the first row of $Y$. These $n$ elements of $\mathbb{C}_{0}^{\prime}$ span a vector space of dimension $q(<n)$ over $K$, the field of constants. Letting $z_{1}, \ldots, z_{q}$ be a basis of this space and letting $z=$ $\left(z_{1}, \ldots, z_{q}, 0, \ldots, 0\right)$, an $n$-tuple with $n-q$ zero entries, there exists $E \in$ $G L(n, K)$ such that $u E=z$. Let $Z$ be the $q \times n$ matrix whose $i$ th row is $D^{i-1} z$. For $s \geqslant 0$ we define $G_{s}$, a $q$-tuple with coefficients in $Q_{0}^{\prime}$, by $s !^{-1} D^{s_{z}}=G_{s} Z$. Estimates for $\left\|G_{s}\right\|_{0}$ are given by Theorem 3.6. Again for $s>0$ let $\alpha_{s}$ be the first row of the matrix $A_{s}$. Let $T$ be the $q \times n$ matrix whose $i$ th row is $(i-1) ! \alpha_{i-1}$. Then (7.1.1) follows from the basic relation $\alpha_{s}=G_{s} T$. This relation may be verified in the form $\alpha_{s} Y E=G_{s} T Y E$, the point being that $\alpha_{s} Y E=s !^{-1} D^{s} u E=s !^{-1} D^{s} z$ and applying 
the same argument to each row of $T Y E$, we recognize that $T Y E=Z$, and so the basic relation follows from the definition of $G_{s}$.

8. Logarithmic singularities. We are concerned here with formalities concerning the transformation (3.7) at a regular singular point. As in $\$ 3$ we take the solutions rather than the differential equation as the basic data.

Let $F_{1}, \ldots, F_{n}$ be elements of $\mathcal{Q}_{0}^{\prime}$, let $\theta$ be an $n \times n$ matrix in Jordan upper triangular normal form and with coefficients in $K$. Let

$$
\left(u_{1}, \ldots, u_{n}\right)=\left(F_{1}, F_{2}, \ldots, F_{n-1}\right) \exp (\theta \log x) \text {. }
$$

For $1<s<n$, let $\theta_{s}$ be the $s \times s$ matrix obtained from $\theta$ by discarding all but the first $s$ rows and columns. Since $\exp (\theta \log x)$ is upper triangular, we may deduce

$$
\left(u_{1}, \ldots, u_{s}\right)=\left(F_{1}, \ldots, F_{s}\right) \exp \left(\theta_{s} \log x\right)
$$

so our formulation is good for truncation. We calculate

$$
D\left(u_{1}, \ldots, u_{n}\right)=\left(\left(F_{1}, F_{2}, \ldots, F_{n-1}\right) E_{\theta}\right) \exp \theta \log x
$$

where $E_{\theta}$ denotes the operation

$$
\left(F_{1}, \ldots, F_{n}\right) \mapsto D\left(F_{1}, \ldots, F_{n}\right)+\frac{1}{x}\left(F_{1}, \ldots, F_{n}\right) \theta
$$

which we write $\left(F_{1}, \ldots, F_{n}\right) E_{\theta}$ with $E_{\theta}$ on the right since the matrix $\theta$ is on the right. Letting $F=\left(F_{1}, \ldots, F_{n}\right)$, the conventional wronskian matrix for $u_{1}, \ldots, u_{n}$ is then given by

$$
\left[\begin{array}{c}
F \\
F E_{\theta} \\
F E_{\theta}^{2} \\
\vdots \\
F E_{\theta}^{n-1}
\end{array}\right] \exp (\theta \log x)
$$

and so the wronskian of $u_{1}, \ldots, u_{n}$ is the product of an element of $Q_{0}^{\prime}$ with the determinant of $\exp \theta \log x$, i.e. with $x^{\operatorname{Tr} \theta}, \operatorname{Tr} \theta$ denoting the trace of $\theta$.

It now follows from (3.10.1.1) that we may write

$$
v_{s+1}=\xi_{s+1} \cdot x^{\lambda_{s+1}-\lambda}
$$

where $\xi_{s+1} \in \mathbb{Q}_{0}^{\prime}$ and $\lambda_{s}$ denotes the $s$ th diagonal entry of $\theta$. Thus aside from a monomial factor involving exponent differences, the $v_{j}$ are meromorphic.

8.6 THEOREM. Let $\theta$ be an $n \times n$ matrix in Jordan normal form with coefficients in $K$ and with all difference of eigenvalues in $\mathbf{Z}_{p}$. Let $F_{1}, \ldots, F_{n}$ be elements of $\mathbb{Q}_{0}^{\prime}$ and let $u_{1}, \ldots, u_{n}$ be given by equation (8.1). We assume that the wronskian of $u_{1}, \ldots, u_{n}$ is not identically zero. Then $u_{1}, \ldots, u_{n}$ span the solution space of an nth order differential equation (3.2) with coefficients in $Q_{0}^{\prime}$ and the estimates (3.6) are valid.

Proof. It follows from (8.5) that each first order factor, $L_{j}$, in (3.7.1) lies in $Q_{0}^{\prime}[D]$. This shows that $L$ lies in the same ring, and hence the $G_{s, j}$ in (3.2) lie in $\mathbb{Q}_{0}^{\prime}$. 
Thus it makes sense to discuss $\left\|G_{s, j}\right\|_{0}$. However this may also be seen directly from (3.11.1) where $G$ is described in terms of quantities such as $A_{s, k}\left(v_{1}, \ldots, v_{k}\right)$. These in turn reduce by the definition ((2.2), (2.7)) to products of terms such as $v_{i}^{\left(\lambda_{1}\right)} /\left(\lambda_{i} ! v_{i}\right)$. The $v_{i}$ being given by (8.5), we know by (1.10) that $v_{i}^{\left(\lambda_{1}\right)} /\left(\lambda_{i} ! v_{i}\right)$ lies in $\mathcal{Q}_{0}^{\prime}$ and since the eigenvalue differences lie in $Z_{p}$, estimate (1.8) is valid. This shows that $A_{s, k}\left(v_{1}, \ldots, v_{k}\right)$ lies in $\mathcal{Q}_{0}^{\prime}$ and that equation (2.8) holds in the present situation. The estimates for $G$ now follow from (3.11.1). This completes the proof of the theorem.

9. Unbounded wronskian. The object of this section is to extend Theorem 4.2 to the case in which $w$ is unbounded on $Q_{0}$. Our method is to systematically replace the estimates for the boundary seminorms, \|\|$_{0}$ in $\$ \$ 2,3$ with estimates for the norm, $\mid l_{0}(r)$ with $r<1$. We list these estimates, adjoining the numeral 9 before the corresponding boundary seminorm estimate.

If $u \in \mathbb{Q}_{0}^{\prime}$ then

$$
\left|\frac{1}{s !} D^{s} u / u\right|_{0}(r)<\frac{1}{r^{s}}
$$

If $v_{1}, \ldots, v_{m} \in Q_{0}^{\prime}$ then

$$
\left|A_{s, k}\left(v_{1}, \ldots, v_{m}\right)\right|_{0}(r) \leqslant\{s, k-1\}_{p} / r^{s+1-k}
$$

and

$$
\left|s ! A_{s, k}\left(v_{1}, \ldots, v_{m}\right)\right|_{0}(r)<1 / r^{s+1-k} .
$$

If $u_{1}, \ldots, u_{n}$ are elements of $\mathcal{Q}_{0}^{\prime}$ with nontrivial wronskian then

$$
\left|G_{s, j}\right|_{0}(r) \leqslant\{s, n-1\}_{p} / r^{s+1-j} \text {. }
$$

Under the hypothesis of (9.3.6), let $v_{1}, \ldots, v_{n}$ be given by (3.7) and $\Gamma$ by (3.12). Then for $1 \leqslant \alpha, \beta \leqslant n$.

$$
\left|\Gamma_{\alpha, \beta}\right|_{0}(r) \leqslant 1 / r^{\alpha-\beta}
$$

and

$$
\left|G_{s, j}\right|_{0}(r) \leqslant \sup _{1<k<n}\left|A_{s, k}\left(v_{1}, \ldots, v_{k}\right)\left(\Gamma^{-1}\right)_{k, j}\right|_{0}(r) .
$$

EXPLANATION AND PROOF. Statement (9.1.8) is a repetition of 1.6. The proof of (9.2.8) is the same as that of (2.8), simply replacing (1.8) by (9.1.8). Equation (9.2.8) implies (9.2.9).

Equation (9.3.6) is proven by following the proof of (3.6), the only changes are that (3.13) and (3.14) must be replaced by (9.3.13) and (9.3.14). Equation (9.3.14) follows from (3.11.1) and (9.3.13) follows from the proof of (3.13) replacing (2.9) by (9.2.9).

9.4.2 TheOREM. Let $u_{1}, \ldots, u_{n}$ be elements of $\mathbb{Q}_{0}$ with nontrivial and possibly unbounded (modified) wronskian $w$. For each $s \in \mathbf{N}$ let

$$
f(s)=\operatorname{Inf}_{r \in(0,1)}|w|_{0}(r) / r^{s}
$$

then for each $u=\Sigma a_{s} x^{s}$ in the $K$-span of $u_{1}, \ldots, u_{n}$ we have the asymptotic estimate

$$
\left|a_{s}\right|=O\left(s^{n-1} f(s)\right)
$$


Proof. We follow the proof of (4.2), but replace (4.2.3): Let $0<\varepsilon<r<1$. We choose $c \in D(0, \varepsilon)$ such that $w(c) \neq 0$.

$$
\left|\left(G_{s, j} \cdot w\right)(c)\right|<\left|G_{s, j} w\right|_{0}(r)=\left|G_{s, j}\right|_{0}(r) \cdot|w|_{0}(r)
$$

It follows from (9.3.6) that

$$
|w(c)|\left|G_{s, j}(c)\right| \leqslant s^{n-1} \varepsilon^{j-1} \operatorname{Inf}_{r \in(\varepsilon, 1)}|w|_{0}(r) / r^{s}
$$

It follows from (1.11.5) that asymptotically

$$
\left|G_{s, j}(c)\right|=O\left(s^{n-1} f(s)\right)
$$

Following the proof of (4.2) this gives the estimates for the coefficients of $u$ ( $\in K$-span of $u_{1}, \ldots, u_{n}$ ) as power series in $(x-c)$. By Lemma 1.11 the same estimates hold for $u$ as power series in $x$. This completes the proof of the theorem.

It seems clear that effective bounds could be found.

\section{REFERENCES}

1. P. Deligne, Équations différentielles à points singuliers réguliers, Lecture Notes in Math., vol. 163, Springer, Berlin and New York, 1970.

2. B. Dwork, Zeta function of hypersurface. II, Ann. of Math. 80 (1964), 227-299.

3. __ p-adic cycles, Publ. Math. Inst. Hautes Etudes Sci., No. 37, 1969.

4. __ On p-adic differential equations. II, Ann. of Math. 98 (1973), 366-376.

5. $\longrightarrow$ On ordinary linear $p$-adic differential equations with algebraic function coefficients, Groupe d'Étude d'Analyse Ultramétrique 1975/76, exposé 18, Secrétariat Math., Paris, 1977.

6. B. Dwork and P. Robba, On ordinary linear p-adic differential equations, Trans. Amer. Math. Soc. 231 (1977), 1-46.

7. G. Frobenius, Ueber der Begriff der Irreductibilität, in Theorie der linear Differentialgleichungen, J. Reine Angew. Math. 76 (1873), 236-270.

7a. (1874), 245-257.

8. N. Katz, Travaux de Dwork, Séminaire Bourbaki 1971/72, Lecture Notes in Math., vol. 317, Springer, Berlin and New York, 1973, pp. 167-200.

9. E. Poole, Introduction to the theory of linear differential equations, Oxford Univ. Press, Oxford, 1936.

10. P. Robba, On the index of p-adic linear differential operators. I, Ann. of Math. 101 (1975), 280-316.

11. __ Nouveaupoint de vue sur le prolongement algébrique, Groupe d'Étude d'Analyse Ultramétrique 1976/77, exposé 5, Secrétariat Math., Paris.

12. Disque générique et équations différentielles, Groupe d'Étude d'Analyse Ultramétrique 1976/77, exposé 8, Secrétariat Math., Paris.

13. P. Robba and B. Dwork, Sur les équations différentielles linéaires p-adiques. II, Trans. Amer. Math. Soc. (to appear).

Department of Mathematics, Princeton University, Princeton, New Jersey 08540

Département de Mathématique, Université de Paris XI, 91400 Orsay, France 